\title{
Ghost Infarct Core and Admission Computed Tomography Perfusion: Redefining the Role of Neuroimaging in Acute Ischemic Stroke
}

\author{
Nuno Martins ${ }^{d} \quad$ Ana Aires $^{\mathrm{e}, \mathrm{f}} \quad$ Beatriz Mendez $^{\mathrm{g}} \quad$ Sandra Boned $^{\mathrm{a}, \mathrm{b}}$ \\ Marta Rubiera $^{a, b} \quad$ Alejandro Tomasello ${ }^{c}$ Pilar Coscojuela ${ }^{c}$ \\ David Hernandez ${ }^{c}$ Marián Muchada $a, b$ David Rodríguez-Luna ${ }^{a, b}$ \\ Noelia Rodríguez ${ }^{a, b}$ Jesús M. Juega ${ }^{a, b}$ Jorge Pagola ${ }^{a, b}$ \\ Carlos A. Molina ${ }^{a, b}$ Marc Ribóa, b \\ aStroke Unit, Department of Neurology, Vall d'Hebron University Hospital, Vall d'Hebron
Research Institute, Barcelona, Spain; bepartament de Medicina, Universitat Autónoma
de Barcelona, Barcelona, Spain; ' 'Department of Neuroradiology, Vall d'Hebron University
Hospital, Barcelona, Spain; d Department of Internal Medicine, Hospital Fernando Fonseca,
Amadora, Portugal; e Department of Neurology, São João Hospital Center, Porto, Portugal;
fDepartment of Clinical Neurosciences and Mental Health, Faculty of Medicine, University of
Porto, Porto, Portugal; 9 Instituto Nacional de Neurología y Neurocirugía, Mexico City, Mexico
}

\section{Keywords}

Acute ischemic stroke - Cerebral blood flow · Computed tomography perfusion .

Endovascular treatment $\cdot$ Ghost core infarct

\begin{abstract}
Background: Determining the size of infarct extent is crucial to elect patients for reperfusion therapies. Computed tomography perfusion (CTP) based on cerebral blood volume may overestimate infarct core on admission and consequently include ghost infarct core (GIC) in a definitive lesional area. Purpose: Our goal was to confirm and better characterize the GIC phenomenon using CTP cerebral blood flow (CBF) as the reference parameter to determine infarct core. Methods: We performed a retrospective, single-center analysis of consecutive thrombectomies of middle cerebral or intracranial internal carotid artery occlusions considering noncontrast CT Alberta Stroke Program Early CT Score $\geq 6$ in patients with pretreatment CTP. We used the RAPID ${ }^{\circledR}$ software to measure admission infarct core based on initial CBF. The final infarct was extracted from follow-up CT. GIC was defined as initial core minus final infarct $>10 \mathrm{~mL}$. Results: A total of 123 patients were included. The median National Institutes of
\end{abstract}

N. Martins and A. Aires contributed equally to this paper. 
Health Stroke Scale score was $18(13-20)$, the median time from symptoms to CTP was 188 (67-288) min, and the recanalization rate (Thrombolysis in Cerebral Infarction score 2b, 2c, or 3 ) was $83 \%$. Twenty patients (16\%) presented with GIC. GIC was associated with shorter time to recanalization (150 [105-291] vs. 255 [163-367] $\mathrm{min}, p=0.05)$ and larger initial CBF core volume (38 [26-59] vs. 6 [0-27] mL, $p<0.001$ ). An adjusted logistic regression model identified time to recanalization $<302 \min (\mathrm{OR} 4.598,95 \% \mathrm{Cl} 1.143-18.495, p=0.032)$ and initial infarct volume (OR 1.01, 95\% Cl 1.001-1.019, $p=0.032$ ) as independent predictors of GIC. At $24 \mathrm{~h}$, clinical improvement was more frequent in patients with GIC (80 vs. $49 \%, p=0.01)$. Conclusions: $\mathrm{CTP}$ CBF $<30 \%$ may overestimate infarct core volume, especially in patients imaged in the very early time window and with fast complete reperfusion. Therefore, the CTP CBF technique may exclude patients who would benefit from endovascular treatment.

(c) 2018 S. Karger AG, Basel

\section{Introduction}

Early arterial recanalization is one of the most important goals to address in the management of acute ischemic stroke in order to save larger hypoperfused areas of parenchyma and to obtain smaller final infarct volumes, which enables better functional outcomes [1-3]. However, inadequate selection of patients for reperfusion therapy may deprive some patients of treatment or offer futile treatment to others. Therefore, determining the size of irreversible infarct core extent on admission is crucial to select the right patients for reperfusion therapies [3].

Multimodal imaging has been proposed to identify the best candidates with large vessel occlusion to receive endovascular reperfusion therapy (EVT) based on mismatch concept [4-6]. Diffusion-weighted imaging (DWI) on MRI is considered the most rigorous method to assess the size of initial infarct core, since it can identify cytotoxic edema within minutes of ischemic injury, with a sensitivity and specificity close to $100 \%[7,8]$. On the other hand, computed tomography perfusion (CTP) is broadly accessible and can be more easily used in emergency settings. Whereas some authors found a good correlation between the initial lesion characterized by decreased cerebral blood volume (CBV) and final infarct [9-12], others such as Bivard et al. [13], considered cerebral blood flow (CBF) a more specific parameter to define infarct core. The best CTP predictor of infarct core is not validated yet, and models using several perfusion measures have been proposed [14, 15].

A recent study from our center showed that CTP CBV may overestimate in some situations infarct core on admission by predicting lesion in areas that will not show infarct on follow-up imaging. The phenomenon was named "ghost infarct core" (GIC) and was associated with early imaging after onset followed by early complete recanalization [15]. In the present study, we aimed to confirm and better characterize this phenomenon using CTP CBF as the reference parameter to determine infarct core.

\section{Methods}

We performed a retrospective (from January 2014 to June 2017), single-center analysis of consecutive patients who received EVT due to proximal middle cerebral artery (M1-MCA) occlusion, terminal internal carotid artery (ICA) occlusion, or tandem extracranial/intracranial occlusion. EVT treatment was indicated if noncontrast CT Alberta Stroke Program Early CT Score (ASPECTS) was $\geq 6$. All patients received CT angiography on admission to determine the presence and location of vessel occlusion and a CTP study, whose results were not considered for treatment decision in patients presenting within $8 \mathrm{~h}$ of symptom onset. 


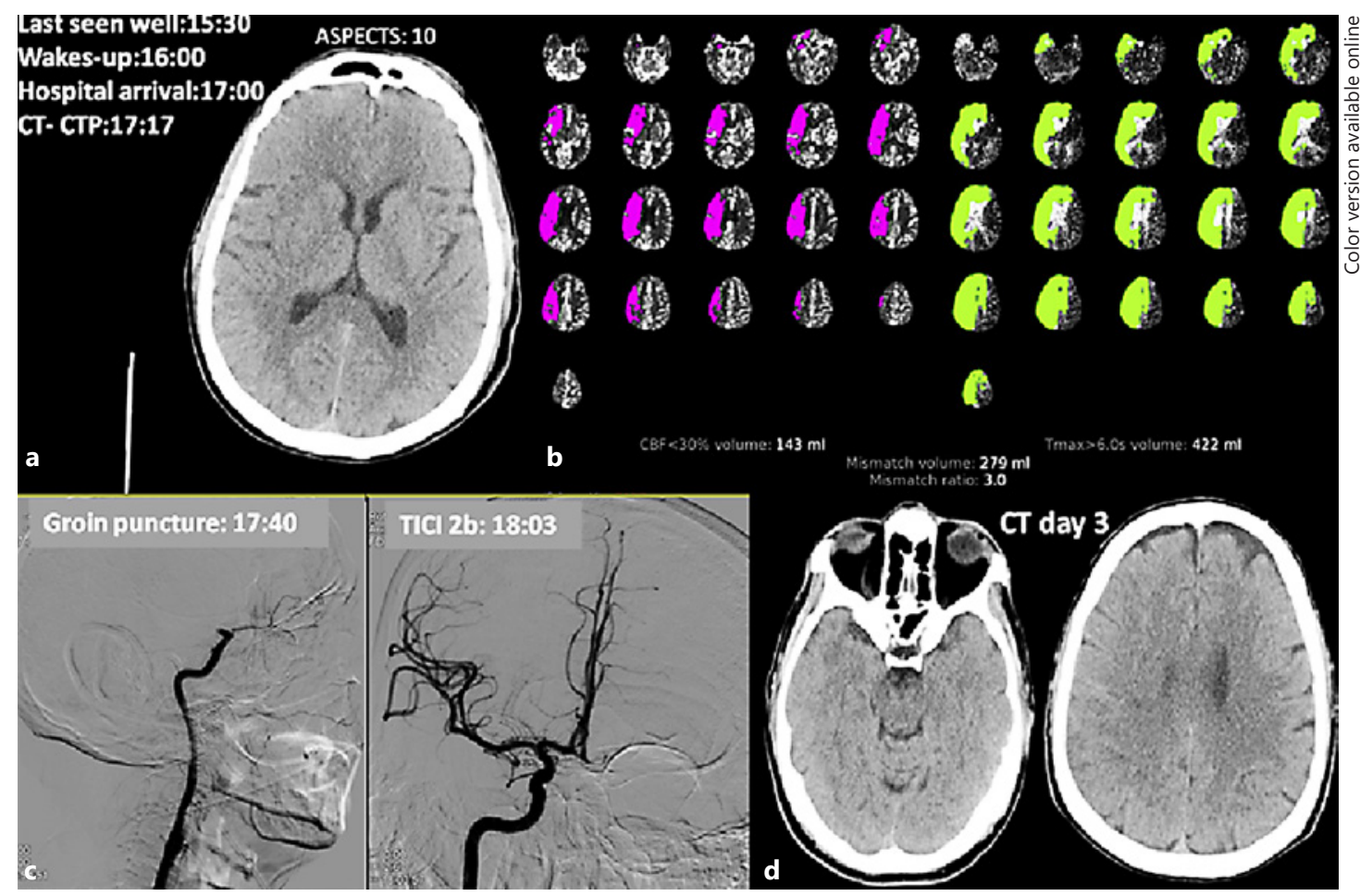

Fig. 1. Admission CT (a), admission CTP with $\mathrm{CBF}<30 \%$ (b), initial and post-revascularization angiography (c), and 72-h CT (d) of a 70-year-old male who presented to the emergency department with signs and symptoms of a right total anterior circulation infarct. The NIHSS score at admission was 19. Brain CT presented blurred outlines of right lentiform nucleus and angio-CT revealed occlusion of right internal carotid artery and initial portion (M1) of the right middle cerebral artery with an infarct core of $143 \mathrm{~mL}$ based on CBF $<30 \%$. The patient underwent endovascular treatment, with a successful Thrombolysis in Cerebral Infarction score $2 \mathrm{~b}$ at first pass. The time from symptom onset to CT was $107 \mathrm{~min}$ and the time to recanalization was $153 \mathrm{~min}$. After the procedure, the NIHSS score improved to 14 and was 5 on day 5 . Follow-up CT on day 3 showed very limited infarct. At 3 months, the modified Rankin Scale score was 2. ASPECTS, Alberta Stroke Program Early CT Score; CBF, cerebral blood flow; CTP, computed tomography perfusion; NIHSS, National Institutes of Health Stroke Scale; TICI, Thrombolysis in Cerebral Infarction.

Endovascular procedures were performed by experienced interventionalists with commercially available thrombectomy devices according to the interventionalists' preferences. Recanalization was assessed and was considered complete if the Thrombolysis in Cerebral Infarction score was 2b, 2c, or 3. The time from symptom onset to CTP and to recanalization was recorded. Final infarct volume was measured on follow-up imaging at $24 \mathrm{~h}$ on noncontrast cerebral tomography by a vascular neurologist using the $\mathrm{A} \times \mathrm{B} \times \mathrm{C} / 2$ formula [16]. GIC was defined and considered when initial core volume minus final infarct volume was $>10 \mathrm{~mL}$ [15] (Fig. 1). Clinical improvement was defined as a decrease of $\geq 4$ points in the National Institutes of Health Stroke Scale (NIHSS) score at $24 \mathrm{~h}$. The modified Rankin Scale (mRS) was used to assess functional outcome at 90 days; a favorable outcome was defined as an mRS score of 0-2.

\section{Imaging Protocol}

CTP was performed on a Definition AS Siemens (Siemens, Erlangen, Germany) 128-section scanner with the following parameters: collimator of $32 \times 1.5 \mathrm{~mm}, 80 \mathrm{kVp}$, and $200 \mathrm{mAs}$ with total coverage of $86 \mathrm{~mm}$. The plane of imaging was parallel to the floor of the anterior cranial fossa starting just above the orbits. Thirty cycles were obtained with a total scan time of $46 \mathrm{~s}$. The images were sent to the RAPID ${ }^{\circledR}$ software (iSchemaView) in order to quantify ischemic core ( $\mathrm{CBF}$ core) as the area with $>70 \%$ reduction in $\mathrm{CBF}(\mathrm{rCBF}<0.3)$ in comparison to the mean $\mathrm{CBF}$ of normally perfused brain parenchyma. 
Table 1. Baseline characteristics

\begin{tabular}{|c|c|c|c|c|}
\hline Variable & $\begin{array}{l}\text { All patients } \\
(n=123)\end{array}$ & $\begin{array}{l}\mathrm{GIC}>10 \mathrm{~mL} \\
(n=20)\end{array}$ & $\begin{array}{l}\text { No GIC } \\
(n=103)\end{array}$ & $\begin{array}{l}p \\
\text { value }\end{array}$ \\
\hline Sex (female/male) & $77 / 46$ & $13 / 7$ & $64 / 39$ & 0.80 \\
\hline Age, years & $78(67-83)$ & $77(64-83)$ & $78(68-83)$ & 0.69 \\
\hline Previous mRS score $\leq 2$ & $99.20 \%$ & $100 \%$ & $99 \%$ & 0.66 \\
\hline Hypertension & $72.40 \%$ & $70 \%$ & $72.80 \%$ & 0.80 \\
\hline Diabetes mellitus & $23.60 \%$ & $25 \%$ & $23.30 \%$ & 0.87 \\
\hline Atrial fibrillation & $43.90 \%$ & $60 \%$ & $40.80 \%$ & 0.11 \\
\hline Hyperlipidemia & $39.80 \%$ & $45 \%$ & $38.80 \%$ & 0.60 \\
\hline Admission glycemia, mg/dL & $124(108-170)$ & $116(107-173)$ & $126(107-173)$ & 0.56 \\
\hline Admission NIHSS score & $18(13-20)$ & $19(16-21)$ & $17(12-20)$ & 0.26 \\
\hline Symptom onset to CTP, min & $188(67-288)$ & $107(50-227)$ & $193(76-294)$ & 0.10 \\
\hline Time from CTP to recanalization, min & $56(40-84)$ & $42(33-78)$ & $63(42-85)$ & 0.13 \\
\hline Symptom onset to recanalization, min & $241(142-352)$ & $150(105-291)$ & $255(163-367)$ & 0.05 \\
\hline Time to CTP $<259$ min & $69 \%$ & $85 \%$ & $65.60 \%$ & 0.09 \\
\hline Time to recanalization $<302 \mathrm{~min}$ & $63.30 \%$ & $84 \%$ & $58.90 \%$ & 0.04 \\
\hline Recanalization rate (TICI score $2 \mathrm{~b}, 2 \mathrm{c}$, or 3 ) & $82.90 \%$ & $90 \%$ & $81.60 \%$ & 0.36 \\
\hline
\end{tabular}

Values are presented as $n / n$, median (IQR), or percentage. CTP, computed tomography perfusion; GIC, ghost infarct core; mRS, modified Rankin Scale; NIHSS, National Institutes of Health Stroke Scale; TICI, Thrombolysis in Cerebral Infarction.

\section{Statistical Analysis}

Descriptive and frequency statistical analyses were obtained using the SPSS v.17.0 software. The Shapiro-Wilk test was used to assure normality of continuous variables. Categorical variables were presented as absolute values and percentages and continuous variables as medians (IQRs) as none was normally distributed. Statistical significance for intergroup differences was assessed by the Pearson $\chi^{2}$ test or the Fisher exact test for categorical variables and by the Mann-Whitney $U$ test for continuous variables. Multivariable logistic regression analyses were used to determine factors that could be considered as independent predictors of favorable outcome. Receiver operating characteristic (ROC) curve analysis was used to calculate the best cutoff time point after which the GIC becomes irrelevant. A probability value of $\leq 0.05$ was considered significant for all tests.

\section{Results}

A total of 123 patients were included; their baseline characteristics are shown in Table 1. The median NIHSS score was 18 (13-20) and the median time from symptoms to CTP was 188 (67-288) min. Complete recanalization was achieved in $80 \%$ in a median time of $67 \mathrm{~min}$ (40-84 min after CTP). Intravenous tissue plasminogen activator was administered in 53 patients (43.4\%) before the endovascular procedure.

Imaging characteristics and outcomes are shown in Table 2.

Occlusion locations were M1-MCA 99 (80.4\%), tandem ICA/MCA 4 (3.3\%), and terminal ICA 20 (16.3\%).

The median CBF core on admission was $8(0-35) \mathrm{mL}$ and the median final infarct volume was 12 (IQR 1-67) mL. In 20 patients (16\%) GIC was identified, and the mean infarct growth in these patients was $-37( \pm 30.7) \mathrm{mL}$. In univariate analysis, GIC was associated with shorter time to recanalization (150 [105-291] vs. 255 [163-367] $\mathrm{min}, p=0.05$ ) as well as larger initial CBF core volume (38 [26-59] vs. 6 [0-27] $\mathrm{mL}, p<0.001$ ) and showed a statistically nonsignificant trend with shorter time to CTP (107 [50-227] vs. 193 [76-294] $\mathrm{min}, p=0.1)$. 
Table 2. Imaging outcomes and lesion volumes

\begin{tabular}{|c|c|c|c|c|}
\hline Variable & $\begin{array}{l}\text { All patients } \\
(n=123)\end{array}$ & $\begin{array}{l}\mathrm{GIC}>10 \mathrm{~mL} \\
(n=20)\end{array}$ & $\begin{array}{l}\text { No GIC } \\
(n=103)\end{array}$ & $\begin{array}{l}p \\
\text { value }\end{array}$ \\
\hline Admission CT ASPECTS score & $9(8-10)$ & $9(7-10)$ & $9(8-10)$ & 0.90 \\
\hline Intravenous tPA pretreatment & $43.40 \%$ & $55 \%$ & $41.20 \%$ & 0.25 \\
\hline Occlusion location & & & & 0.84 \\
\hline M1-MCA & $80.40 \%$ & $80 \%$ & $80.60 \%$ & \\
\hline Terminal ICA & $16.30 \%$ & $15 \%$ & $16.50 \%$ & \\
\hline Tandem ICA/MCA & $3.30 \%$ & $5 \%$ & $2.90 \%$ & \\
\hline TOAST & & & & 0.39 \\
\hline Atherothrombotic & $2.40 \%$ & $5 \%$ & $1.90 \%$ & \\
\hline Cardioembolic & $71.50 \%$ & $85 \%$ & $68.90 \%$ & \\
\hline Undetermined & $20.30 \%$ & $5 \%$ & $23.30 \%$ & \\
\hline Other determined (dissections) & $5.70 \%$ & $5 \%$ & $5.80 \%$ & \\
\hline CBF core, mL & $8(0-35)$ & $38(26-59)$ & $6(0-27)$ & 0.00 \\
\hline Final infarct volume, mL & $12(1-67)$ & $6(1-13)$ & $15(1-80)$ & 0.05 \\
\hline Infarct growth, mL & $3.56(-5$ to 34$)$ & $-27(-48.4$ to -14$)$ & $7(0-50)$ & 0.00 \\
\hline
\end{tabular}

Values are presented as median (IQR) or percentage. ASPECTS, Alberta Stroke Program Early CT Score; $\mathrm{CBF}$, cerebral blood flow; GIC, ghost infarct core; ICA, internal carotid artery; M1-MCA, proximal middle cerebral artery; MCA, middle cerebral artery; tPA, tissue plasminogen activator.

Fig. 2. Infarct growth (final infarct volume minus initial core) and time to recanalization. The red line represents time of 302 min. GIC, ghost infarct core.

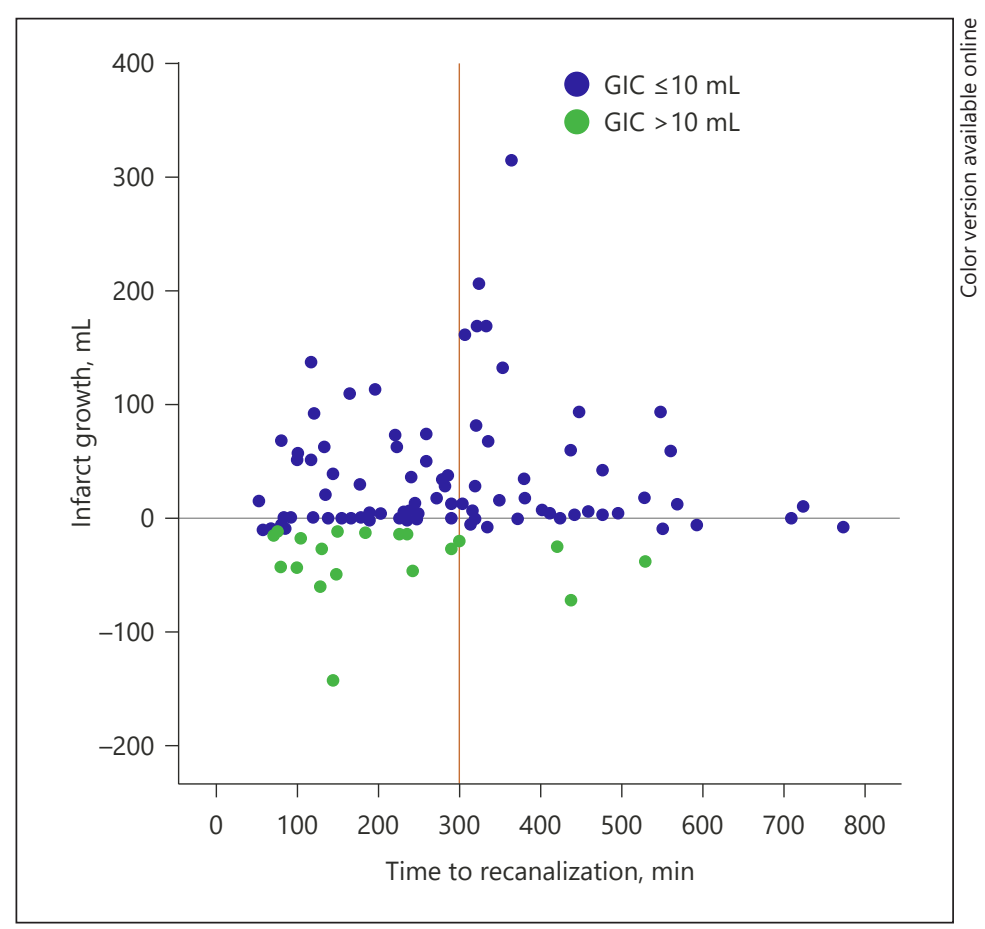

ROC curve analysis showed a cutoff point of $302 \mathrm{~min}$ in time to recanalization (S: 41.1\%; E: $84.2 \%$ ) to be associated with the presence of GIC.

Logistic regression adjusting for time to recanalization $<302 \mathrm{~min}$ and $\mathrm{CBF}$ core found both time to recanalization $<302 \min$ (OR 4.598, 95\% CI 1.143-18.495, $p=0.032$ ) and initial infarct volume (OR 1.01, 95\% CI 1.001-1.019, $p=0.032$ ) to be independent predictors of GIC.

Figure 2 shows infarct evolution according to time to recanalization. 
Table 3. Clinical outcomes

\begin{tabular}{lcccc}
\hline Variable & $\begin{array}{c}\text { All patients } \\
(n=123)\end{array}$ & $\begin{array}{l}\text { GIC }>10 \mathrm{~mL} \\
(n=20)\end{array}$ & $\begin{array}{l}\text { No GIC } \\
(n=103)\end{array}$ & $p$ value \\
\hline NIHSS score at 24h & $10(3-18)$ & $8(4-12)$ & $11(3-18)$ & 0.35 \\
NIHSS score improvement at 24 h & $5(0-11)$ & $11(6-15)$ & $3(0-9)$ & 0.00 \\
Clinical improvement & $53.70 \%$ & $80 \%$ & $48.5 \%$ & 0.01 \\
mRS score $\leq 2$ at 90 days & $36.80 \%$ & $52.60 \%$ & $31.10 \%$ & 0.12 \\
Hemorrhagic transformation & $20.30 \%$ & $20 \%$ & $20.40 \%$ & 0.97 \\
In-hospital death & $7.50 \%$ & $5.30 \%$ & $7.90 \%$ & 0.69 \\
\hline
\end{tabular}

Values are presented as median (IQR) or percentage. GIC, ghost infarct core; mRS, modified Rankin Scale; NIHSS, National Institutes of Health Stroke Scale.

As theoretically complete recanalization is a necessary to observe the GIC phenomenon, we repeated the analysis including only those patients with complete recanalization $(n=$ 102). Of those, $18(17.6 \%)$ presented GIC. GIC was associated with time to recanalization (150 [117-267] vs. 245 [165-365], $p=0.04$ ) and showed a statistically nonsignificant trend with shorter time to CTP (107 [48-216] vs. 192 [77-296], $p=0.08)$. ROC curve analysis showed cutoff points in time from symptom onset to CTP $<259$ min (time to CTP $<259 \mathrm{~min}$ : GIC $=$ $23.2 \%$ vs. time to $\mathrm{CTP} \geq 259 \mathrm{~min}$ : $\mathrm{GIC}=6.7 \%, p=0.05$ ) and time from onset to recanalization $<302 \mathrm{~min}$ (time to recanalization $<302 \mathrm{~min}$ : $\mathrm{GIC}=24.6 \%$ vs. time to recanalization $\geq 302 \mathrm{~min}$ : $\mathrm{GIC}=6.5 \%, p=0.03$ ) to be associated with presence of GIC.

Logistic regression adjusting for time to CTP $<259 \mathrm{~min}$, time to recanalization $<302 \mathrm{~min}$, and CBF core found only time to recanalization $<302 \mathrm{~min}$ (OR $0.33,95 \%$ CI $0.002-0.686, p=$ 0.03 ) and initial infarct volume (OR 1.02, 95\% CI 1.003-1.027, $p=0.01$ ) to be independent predictors of GIC.

Clinical outcomes are shown in Table 3.

At $24 \mathrm{~h}$, clinical improvement (53.7\% overall) was more frequent in patients with GIC (80 vs. $49 \%, p=0.01$ ) as compared with patients without GIC. Favorable outcome at 90 days was achieved in 45 patients (36.8\%) with a statistically nonsignificant trend favoring GIC patients (52.60 vs. $31.10 \%, p=0.1$ ). Logistic regression adjusting for GIC and CBF core showed GIC to be an independent predictor of favorable outcome at 90 days (OR 3.019, 95\% CI 1.014-8.989, $p=0.05)$.

\section{Discussion}

Since endovascular procedures are considered a stroke treatment, the presence of salvageable brain tissue and the decision to treat patients take into account not only established ischemic core, but also mismatch concept - the difference between ischemic tissue at risk and the already irreversibly infarcted area. Therefore, an effort has been made to find a highly specific method to depict ischemic penumbra from established infarct in the first hours after symptom onset. Considering that current technology achieves high recanalization rates, the observed mismatch area would be saved from infarct [17-19]. Some authors found a good correlation between the initial lesion characterized by decreased CBF or CBV and final infarct volume $[18,20,21]$. However, a recent study from our center introduced the concept of GIC. GIC is defined as the infarct core initially predicted by CTP that appears as intact brain tissue on follow-up imaging. The existence of GIC implies that the initial core estimated by CTP may 
include, to some extent, salvageable brain tissue [15]. Characterization of this phenomenon is crucial to avoid exclusion of patients from a treatment that can still be of benefit.

In this study, we aimed to evaluate the presence of GIC using CBF to identify initial infarct core on admission. We applied a widely used automated imaging software to generalize our results. The percentage of patients with GIC was $16 \%$, a value lower than that observed in our previous work using CBV (38\%). This observation reflects a slightly lower but still significant overestimation of infarct core on admission if CBF is used instead of CBV. Figure 1 shows a perfect example of a patient with a large infarct core that did not have correspondence on the 24-h follow-up noncontrast CT. According to our results, the significant amount of GIC is determined by the fact that the patient was imaged very early after symptom onset and complete recanalization was achieved shortly after. If this patient had not been treated with EVT as suggested by the CTP findings, it is very likely that the outcome would have been worse. Other publications reported similar examples [22].

Our results reinforce the notion that GIC is time dependent. CBF is a hemodynamic measurement that represents the rate at which contrast (or blood) flows through brain tissue. This measurement correlates on average, but with exceptions, with infarct core. Opposed to CTP, DWI is able to show actual tissular changes related with cellular death, such as cytotoxic edema. Initially the observed CTP lesion seems to still be reversible; however, as time goes by, the persistence of the occlusion will precipitate irreversible tissue damage. Therefore, the capacity of CTP to predict initial infarct core may depend on both time to imaging and time to recanalization. In this study, we found a cutoff of approximately $4.5 \mathrm{~h}$ to imaging and of $5 \mathrm{~h}$ to recanalization as independent predictors of GIC in CBF $<30 \%$. These results are in line with our previous findings [15] and others [9].

Previous studies supporting CTP as a robust predictor of final infarct did not include patients imaged in the very early time window with a very high degree of complete recanalization rates [20]. As the latest advances in stroke treatment suggest, we are facing a scenario in which these situations will become very frequent. Moreover, current guidelines also recommend to reduce as much as possible all workflow times [23]. In this context, it is important to warn about the GIC phenomenon in order not to exclude patients who could potentially benefit from EVT.

Our results showed that most patients who finally presented GIC had the high ASPECTS on admission despite presumed large infarct cores on CTP, suggesting that in this early time window, patient selection according to noncontrast CT might avoid wrong exclusions from EVT [24].

Angermaier et al. [9] pointed out that when early recanalization is achieved, the initial ASPECTS CTP lesion may overestimate the final infarct, which is in line with previous findings from our center. Our present results also corroborate this theory, since GIC patients have shorter time from symptom onset to CTP, shorter time from CTP to reperfusion, and higher rates of recanalization.

Some studies point to $\mathrm{CBF}$ as being more accurate than CBV in defining infarct core [25]. Moreover, relative CBF values performed significantly better than absolute values in predicting final infarct [26]. In this work, we used relative CBF values and still found a significant incidence of GIC. This may be explained by several facts associated with the technique. One is that CBF definition is dependent on contrast bolus intensity. On the other hand, a short acquisition time may not register the complete transit of contrast bolus, overestimating once more the volume of infarct core [26]. However, our acquisition protocol lasted for $46 \mathrm{~s}$, which was substantially longer compared to others.

A possible way to increase CTP accuracy is to redefine or individualize CBF thresholds according to patient characteristics or time from onset. The RAPID ${ }^{\circledR}$ software classified parenchyma with $\mathrm{CBF}<30 \%$ as infarct core. We may hypothesize that a lower value of relative 
CBF could depict more accurately core and penumbra areas in the very early time window. This hypothesis is being evaluated in a parallel study.

One limitation of our study is the measurement of final infarct volume on noncontrast CT at $24 \mathrm{~h}$. A follow-up DWI might have been more accurate, but for many reasons, including costs and patient tolerance, CT is routinely used for this purpose in our institution. Our results are however validated by the fact that GIC is associated with better clinical outcome. Despite showing significantly larger "presumed" initial infarct core volume, patients with GIC experienced significantly greater NIHSS score improvement at $24 \mathrm{~h}$ and higher rates of favorable outcome mRS score at 90 days. Also, it is possible that the real number of GICs may be higher as some patients with a large presumed infarct core and no mismatch might have been excluded from EVT.

\section{Conclusion}

CTP $\mathrm{CBF}<30 \%$ may overestimate infarct core volume, especially in patients imaged in the very early time window and with fast complete reperfusion. Since under these circumstances infarct core may include salvageable brain tissue, selecting patients for EVT based on the absence of mismatch may deny treatment to patients who would potentially benefit from it.

\section{Disclosure Statement}

The authors have no conflicts of interest to declare.

\section{References}

1 Jovin TG, Chamorro A, Cobo E, de Miquel MA, Molina CA, Rovira A, et al: Thrombectomy within 8 hours after symptom onset in ischemic stroke. N Engl J Med 2015;372:2296-2306.

2 Ribo M, Tomasello A, Lemus M, Rubiera M, Vert C, Flores A, et al: Maximal admission core lesion compatible with favorable outcome in acute stroke patients undergoing endovascular procedures. Stroke 2015;46:2849_ 2852.

3 Skagen K, Skjelland M, Russell D, Jacobsen EA: Large-vessel occlusion stroke: effect of recanalization on outcome depends on the National Institutes of Health Stroke Scale score. J Stroke Cerebrovasc Dis 2015;24: 1532-1539.

4 Aronsson M, Persson J, Blomstrand C, Wester P, Levin LA: Cost-effectiveness of endovascular thrombectomy in patients with acute ischemic stroke. Neurology 2016;86:1053-1059.

5 Bracard S, Ducrocq X, Mas JL, Soudant M, Oppenheim C, Moulin T, et al: Mechanical thrombectomy after intravenous alteplase versus alteplase alone after stroke (THRACE): a randomised controlled trial. Lancet Neurol 2016;15:1138-1147.

6 Mocco J, Zaidat 00, von Kummer R, Yoo AJ, Gupta R, Lopes D, et al: Aspiration thrombectomy after intravenous alteplase versus intravenous alteplase alone. Stroke 2016;47:2331-2338.

7 Copen WA: Multimodal imaging in acute ischemic stroke. Curr Treat Options Cardiovasc Med 2015;17:368.

8 Olivot JM, Sissani L, Meseguer E, Inoue M, Labreuche J, Mlynash M, et al: Impact of initial diffusion-weighted imaging lesion growth rate on the success of endovascular reperfusion therapy. Stroke 2016;47:2305-2310.

9 Angermaier A, Khaw AV, Kirsch M, Kessler C, Langner S: Influence of recanalization and time of cerebral ischemia on tissue outcome after endovascular stroke treatment on computed tomography perfusion. J Stroke Cerebrovasc Dis 2015;24:2306-2312.

10 Lum C, Ahmed ME, Patro S, Thornhill R, Hogan M, Iancu D, et al: Computed tomographic angiography and cerebral blood volume can predict final infarct volume and outcome after recanalization. Stroke 2014;45: 2683-2688.

11 Parsons MW, Pepper EM, Chan V, Siddique S, Rajaratnam S, Bateman GA, et al: Perfusion computed tomography: prediction of final infarct extent and stroke outcome. Ann Neurol 2005;58:672-679.

12 Schramm P, Schellinger PD, Klotz E, Kallenberg K, Fiebach JB, Külkens S, et al: Comparison of perfusion computed tomography and computed tomography angiography source images with perfusion-weighted imaging and diffusion-weighted imaging in patients with acute stroke of less than 6 hours' duration. Stroke 2004;35:1652-1658. 
13 Bivard A, Spratt N, Levi C, Parsons M: Perfusion computer tomography: imaging and clinical validation in acute ischaemic stroke. Brain 2011;134(Pt 11):3408-3416.

14 Bivard A, Levi C, Krishnamurthy V, Hislop-Jambrich J, Salazar P, Jackson B, et al: Defining acute ischemic stroke tissue pathophysiology with whole brain CT perfusion. J Neuroradiol 2014;41:307-315.

15 Boned S, Padroni M, Rubiera M, Tomasello A, Coscojuela P, Romero N, et al: Admission CT perfusion may overestimate initial infarct core: the ghost infarct core concept. J Neurointerv Surg 2017;9:66-69.

16 Sims JR, Gharai LR, Schaefer PW, Vangel M, Rosenthal ES, Lev MH, et al: ABC/2 for rapid clinical estimate of infarct, perfusion, and mismatch volumes. Neurology 2009;72:2104-2110.

17 Bhogal P, Bucke P, Ganslandt O, Bazner H, Henkes H, Perez MA: Mechanical thrombectomy in patients with M1 occlusion and NIHSS score $\leq 5$ : a single-centre experience. Stroke Vasc Neurol 2016;1:165-171.

18 Campbell BC, Mitchell PJ, Yan B, Parsons MW, Christensen S, Churilov L, et al: A multicenter, randomized, controlled study to investigate EXtending the time for Thrombolysis in Emergency Neurological Deficits with Intra-Arterial therapy (EXTEND-IA). Int J Stroke 2014;9:126-132.

19 Saver JL, Goyal M, Bonafe A, Diener HC, Levy EI, Pereira VM, et al: Stent-retriever thrombectomy after intravenous t-PA vs. t-PA alone in stroke. N Engl J Med 2015;372:2285-2295.

20 Benson J, Payabvash S, Salazar P, Jagadeesan B, Palmer CS, Truwit CL, et al: Comparison of CT perfusion summary maps to early diffusion-weighted images in suspected acute middle cerebral artery stroke. Eur J Radiol 2015;84:682-689.

21 Qiao Y, Zhu G, Patrie J, Xin W, Michel P, Eskandari A, et al: Optimal perfusion computed tomographic thresholds for ischemic core and penumbra are not time dependent in the clinically relevant time window. Stroke 2014; 45:1355-1362.

22 Mokin M, Levy EI, Saver JL, Siddiqui AH, Goyal M, Bonafe A, et al: Predictive value of RAPID assessed perfusion thresholds on final infarct volume in SWIFT PRIME (Solitaire With the Intention for Thrombectomy as Primary Endovascular Treatment). Stroke 2017;48:932-938.

23 Fiehler J, Cognard C, Gallitelli M, Jansen O, Kobayashi A, Mattle HP, et al: European Recommendations on Organisation of Interventional Care in Acute Stroke (EROICAS). Int J Stroke 2016;11:701-716.

24 Demeestere J, Garcia-Esperon C, Garcia-Bermejo P, Ombelet F, McElduff P, Bivard A, et al: Evaluation of hyperacute infarct volume using ASPECTS and brain CT perfusion core volume. Neurology 2017;88:2248-2253.

25 Kamalian S, Kamalian S, Maas MB, Goldmacher GV, Payabvash S, Akbar A, et al: CT cerebral blood flow maps optimally correlate with admission diffusion-weighted imaging in acute stroke but thresholds vary by postprocessing platform. Stroke 2011;42:1923-1928.

26 Campbell BC, Christensen S, Levi CR, Desmond PM, Donnan GA, Davis SM, et al: Cerebral blood flow is the optimal CT perfusion parameter for assessing infarct core. Stroke 2011;42:3435-3440. 